\title{
Der Übergang zur Marktwirtschaft - Ein soziokulturelles Problem
}

\author{
Von Hans G. Nutzinger
}

\section{Einleitung}

Reichlich drei Jahre nach dem Zusammenbruch der kommunistisch-planwirtschaftlichen Regime in den Ländern Mittel- und Osteuropa sieht die wirtschaftliche Lage in nahezu allen RGW-Staaten, gegen alle Hoffnungen und Erwartungen im Frühjahr 1990, weitaus schlechter aus, als dies Wirtschaftstheroretiker und Wirtschaftspraktiker vorhergesehen haben, und zwar unabhängig davon, welche ökonomische Politik sie bei der Transformation von der Plan- zur Marktwirtschaft eingeschlagen haben.' An die Stelle der erwarteten blühenden Ostmärkte sind politisch instabile und teilweise wirtschaftich kaum lebensfähig erscheinende Gebilde getreten; nach dem Zusammenbruch der Sowjetunion hat sich die Anzahl der Nationalstaaten in Mittel- und Osteuropa verdreifacht. Diese politischen Umwälzungen waren kaum erwartet und auf jeden Fall gravierend unterschätzt worden; sie haben aber zweifelsfrei mit zu den Erschütterungen des ökonomischen Systems beigetragen, die dann ihrerseits die Konsolidierung der staatlichen Ordnungen verhindert oder zumindest erschwert haben.

Eine der gängigen Erwartungen des Frühjahrs 1990 war, daß sich in den ehemaligen RGW-Ländern - nach dem Abwerfen der planwirtschaftlich-bürokratischen Fesseln und der politischen Bevormundung - die vorhandenen oder zumindest vorhanden geglaubten Wachstumskräfte quasi naturwüchsig Geltung verschaffen würden. Die vorherrschenden Hoffnungen des Westens vor wenig mehr als drei Jahren waren auf einen generellen Aufschwung im Osten gerichtet.

Tatsächlich eingetreten ist so ziemlich genau das Gegenteil der damaligen Erwartungen: Wie die Tabelle zeigt, ist das Sozialprodukt der meisten RGW-Länder im Zeitraum von 1989 (dem letzten Jahr der Planwirtschaft) bis 1992 in einer Größenordnung zwischen 20 und 50\% geschrumpft. Diese Schrumpfung des Bruttosozialprodukts oder Bruttoinlandsprodukts ist in so gut wie allen diesen Volkswirtschaften mit einer Reihe weiterer sozial und wirtschaftlich höchst unerwünschter Folgen verbunden:

- Die registrierte A rbeitslosigkeit, zuvor praktisch in diesen Ländern so gut wie überhaupt nicht bekannt. hat in den letzten drei Jahren dramatisch zugenommen und in einigen Ländern bereits die 10\%-Marke erreicht oder sogar schon überschritten. Diese Größenordnung deutet darauf hin. daß es sich hier um weit mehr als den notwendigen Abbau früherer Uberbeschäftigung, also die Transformation versteckter in offene Arbeitslosigkeit handelt.

- Die Freigabe vieler Preise, eine unverzichtbare Voraussetzung für den Übergang zur Marktwirtschaft. hat weniger zu einer erwünschten Ausweitung der Produktion als vielmehr in den meisten dieser Länder zu gefährlichen inflationären Prozessen, bis hin zu allgemeiner Teuenung und galoppierender Geldentwertung geführt. (Die Erfahrungen mit der Währungsreform 1948 und der damit verbundenen 
Tabelle: Veränderungen der Produktion zwischen 1989 und 1992

\begin{tabular}{ll}
\hline Land & $\begin{array}{l}\text { Produktion }(1992 / 1989 ; \\
\text { in Prozent) }(1989=100)\end{array}$ \\
\hline Albanien & 49 \\
Bulgarien & 63 \\
Tschechien und Slowakei & 78 \\
Deutschland (neue Bundesländer) & 64 \\
Ungarn & 83 \\
Polen & 81 \\
Rumänien & 79 \\
RuBland & 72 \\
Ukraine & 71 \\
\hline
\end{tabular}

Anmerkung: Die Zahlen beziehen sich auf das Bruttosozial- oder das Bruttoinlandsprodukt, bis auf die Ukraine, für die sich die Angaben auf das System der Berechnung materieller Produktionswerte bezieht. Die Zahlen für 1992 sind vorläufige Schätzungen.

Quelle: $\quad$ Stat. Anhang des Weltentwicklungsberichts der Weltbank 1992; leicht verändert nach Fischer (1993, S. 395).

Freigabe vieler Preise in den westlichen Besatzungszonen Deutschlands verlief seinerzeit ganz anders, nämlich viel günstiger und im großen und ganzen genau im Sinne dessen, was die liberale ökonomische Theorie prognostiziert hatte. Auf die Gründe dafür, warum sich die positiven Erfahrungen mit der westdeutschen Währungsreform nach dem Zweiten Weltkrieg nicht auf die Transformation der ehemaligen RGW-Lānder übertragen lieBen, gehe ich später in Abschnitt 3 noch ein.)

- Die Versuche der meisten sogenannten "Reformstaaten Mittel- und Osteuropas», Zölle und nichttarifäre Handelshemmnisse abzubauen, die heimischen Währungen im Verkehr mit anderen Ländern wenigstens teilweise zu liberalisieren, sich auf die Anforderungen und Chancen des Weltmarktes einzustellen und sich damit sinnvoll in die internationale Arbeitsteilung einzugliedern, sind bisher wenig erfolgreich gewesen. Einer der Gründe für den bisherigen Mißerfolg der Bestrebungen ist allerdings darin zu sehen, da $B$ die führenden Industrienationen, insbesondere die Staaten der $E G$ und die USA, ihre Mărkte häufig gerade in den Bereichen (zum Beispiel Stahl oder Agrarprodukte) gegen Importe abgeschlossen haben, in denen die ehemaligen RGW-Länder erfolgreich hätten konkurrieren können. Ein anderer - sozusagen systemspezifischer - Grund dafür besteht aber darin, daB die Umstellung auf dem Weltmarkt - gerade dann, wenn sie vom Ausgangspunkt eines relativ isolierten und rückständigen Austauschs erfolgen mußte, wie er innerbalb des RGW üblich war - in aller Regel erheblich mehr Zeit, Qualifikation, Kapital und Expertise braucht, als man sich im Frühjahr 1990 vorzustellen vermochte. Hinzu $\mathrm{kam}$, daB der Zollabbau die aus anderen Gründen (wirtschaftliche Schrumpfung, Arbeitslosigkeit usw.) ohnehin schon sehr schwierige Situation der öfentlichen Haushalte in diesen Ländern weiter verschärfte2.

Obwohl die Situation in den verschiedenen Reformstaaten durchaus im einzelnen differiert und obgleich sie sich in einigen Ländern, wie der ehemaligen DDR und in Ungarn ${ }^{3}$, jetzt zum Besseren zu wenden scheint, besteht doch immer noch reichlich Grund zu der Befürchtung, daß die schwierige wirtschaftliche Lage - insbesondere die immer noch wachsende Arbeitslosigkeit zu einer politisch-gesellschaftlichen Reformblockade führt, die ihrerseits den erforderlichen wirtschaftlichen Aufschwung verhindert. In gewisser Weise läBt sich die wirtschaftliche Situation 
der meisten Reformstaaten, selbstverständlich mit aller gebotenen Differenzierung im Detail, durchaus mit der Weltwirtschaftskrise in den Jahren 1929-1934 vergleichen: Der Preismechanismus ist nicht in der Lage, binnen kurzer Frist und aus sich allein heraus die notwendigen Anpassungsvorgänge zu initiieren, die für eine makroökonomische Stabilisierung erforderlich wären. Vielmehr sieht es in vielen Ländern so aus, daß sich die wesentlichen makroökonomischen Probleme dieser Volkswirtschaften - wie Schrumpfung des Sozialprodukts, wachsende Arbeitslosigkeit. eine inferiore Position am Weltmarkt und eine Auslandsverschuldung, deren Bedienung die wirtschaftliche Leistungskraft der meisten RGW-Länder deutlich überschreitet wechselseitig verstärken, statt sich irgendswie auszugleichen. Da es hier aber um weit mehr als die Kompensation einer gesamtwirtschaftlichen Nachfragelücke geht, reichen keynesianische Rezepte mit Sicherheit nicht aus. Wir wollen daher die Situation in den neuen Bundesländern und den Reformstaaten Mittel- und Osteuropas etwas genauer betrachten.

\section{Die ehemalige DDR - nicht nur ein Sonderfall}

Wenden wir uns nun dem Beispiel der ehemaligen DDR zu, zum einen, weil sie in mancher Hinsicht einen Sonderfall innerhalb dieser Ländergruppe darstellt, zum anderen aber auch deswegen, weil einige unserer sehr unmittelbaren Erfahrungen mit den neuen Bundesländern durchaus auch einen Erklärungswert für die Probleme der anderen RGW-Staaten besitzen. Nicht ohne Berechtigung hatten viele westliche Experten die wirtschaftliche Leistungsfähigkeit der DDR im Vergleich zu den anderen RGW-Ländern besonders hoch eingestuft. Als weitere günstige Bedingung für einen raschen Aufschwung erschien, daß dieses Land nach seiner Vereinigung mit den westlichen Landesteilen (die allerdings viele Züge eines "Anschlusses" hatte) anders als alle übigen Reformstaaten einen "starken Bruder" (oder vielmehr einen wreichen Onkel () im eigenen Hause hatte, der auch bereit und in der Lage war, in erheblichem Umfange unentgeltliche Hilfe und Unterstützung zu gewähren. Gleichwohl zeigt Tabelle 1, daß gerade Ostdeutschland unter den entwickelteren RGW-Ländern (also insbesondere im Vergleich zu Ungarn, zur Tschechischen und Slowakischen Republik und zu Polen) außerordentlich schlecht abschneidet und, jedenfalls was die Schrumpfung der Produktion betriff, noch hinter RuBland und der Ukraine und auf dem gleichen Niveau wie Bulgarien rangiert. Die Gründe für dieses scheinbare Paradoxon sind leicht zu erkenenn und auch schon ausgiebig in der Literatur erörtert:

- Die wirtschaftliche und politische Vereinigung des Jahres 1990 hat, zusammen mit dem zugrunde gelegten Umtauschsatz, die ostdeutschen Produkte einer Verdrängungskonkurrenz durch die westdeutschen Anbieter ausgesetzt, die weit über qualitätsbedingte Nachteile der DDR-eigenen Produktion hinausging. Aus diesem Grunde vor allem konnte sich auch die Alt-Bundesrepublik in den Jahren 1990 und 1991 von der schon damals beobachteten weltweiten Rezession in den meisten Industrieländern abkoppeln: Die Eroberung des ostdeutschen Marktes wurde in einem doppelten Sinne zu einem whausgemachten Konjunkturprogramm«.

- Dieses hausgemachte Konjunkturprograrım erwies sich zugleich aber auch als in bedeutendes whausgemachtes Problemu: Der dramatische Rückgang der heimischen Produktion in der bald nicht mehr real existierenden DDR bedeutete, wenn auch zeitverzögert, einen zunehmenden Verlust an Arbejtsplätzen, und zwar weit über jenes $\mathrm{MaB}$ hinaus, das durch dii Umwandlung der zuvor 
"versteckten" in woffene " Arbeitslosigkeit - also den Abbau von überflüssiger Beschäftigung zur Effizienzsteigerung - ohnehin schon geboten war. Damit ergaben sich gravierende wirtschaftliche und soziale Verwerfungen.

Der "Vorteile dieser raschen politischen und wirtschaftlichen Vereinigung der DDR mit der AltBundesrepublik - nicht zuletzt die Übernahme einer fest etablierten, weltweit anerkannten und relativ stabilen Währung. der DM - war unter den gegebenen innen- und außenpolitischen Bedingungen mit erheblichen Nachteilen erkauft:

- Die Schaffung eines einheitlichen Staats- und Wirtschaftsgebietes, aufgrund politischer Vorgaben innerhalb kürzester Frist, gab den politischen und ökonomischen Kräften, die auf eine sehr rasche Angleichung aller Güter- und Faktorpreise drängten, ein weitaus stärkeres Gewicht, als dies traditionellen Preisangleichungstendenzen im Rahmen des internationalen Handels zugekommen wäre. Dies läßt sich beispielsweise an der Frage einiger besonders wichtiger Faktorpreise, nämlich der Lōhne und Gehälter, verdeutlichen: Die Tarifvereinbarungen für die neuen Bundesländer in den Jahren 1990 und 1991 wurden weitgel.and auf dem Wege von "Stellvertreterverhandlungen « erzielt, weil es noch keine schlagkräftigen Arbeitnehmer- und Arbeitgeberorganisationen vor Ort gab; statt dessen operierten weitgehend westdeutsche "Stellvertreter" auf beiden Seiten, die, wenn auch aus unterschiedlichen Gründen, eher daran interessiert waren, sich eine Billiglohnkonkurrenz aus den neuen Bundesländern vom Leibe zu halten, als deren wirtschaftlichen Aufschwung lohnpolitisch abzusichern, Da die - im Prinzip sicher langfristig notwendigen - Lohnanpassungen an das westdeutsche Niveau über einen viel rascheren Zeitraum vereinbart wurden, als es dem sehr viel stärker nachhinkenden Produktivitätswachstum in den neuen Bundesländern entsprach, wurden nicht wenige Betriebe auch über Arbeitsentgeite, die nicht der Produktivität entsprachen, aus dem Markt gedrängt und, was noch viel bedenklicher ist: Das Entstehen neuer Betriebe, vor allem im mittelständischen Bereich, und die erwartete Ausweitung der Arbeitsplätze im Bereich des Handwerks unterblieben auch aus diesem Grunde oder wurden jedenfalls ernsthaft dadurch behindert. Zwangslāufig mußte dies die Schrumpfung der industriellen Produktion und die Arbeitsplatzverluste in den neuen Bundeslāndern weiter verschärfen.

Selbstverständlich müssen in einem einheitlichen Staats- und Wirtschaftsgebiet, das sich den Grundgedanken der "Sozialen Marktwirtschaft" verpflichtet weiß, die Gewinne der "Sieger « - wie Eroberung des ostdeutschen Absatzmarktes, Zustrom relativ hochqualifizierter und relativ kostengünstiger Arbeitskräfte aus den neuen Bundesländern, Niederhaltung einer potentiellen ostdeutschen Konkurrenz - teuer bezahlt werden, derzeit mit einem jährlichen Finanztransfer aus öffentlichen Haushaltsmitteln pro Einwohner der neuen Bundesländer in Hōhe von rund 8000 bis 10000 DM (je nach Berechnungsgrundlage) und damit in erster Linie vom westdeutschen Steuerzahler. Wenn man also in aggressiven militärischen oder absatzstrategischen Kategorien denkt, muß man sagen: Der "Sieg « der westdeutschen Anbieter und Tarifpartner über aktuelle und potentielle ostdeutsche Konkurrenten erweist sich als - extrem kostspieliger - Pyrrhussieg, den bekanntermaßen nicht der Verlierer, sondern der Gewinner zu bezahlen hat. Allerdings wird die "Zahllast" des "westdeutschen Gewinners" noch immer wesentlich dadurch erleichtert, da $B$ ein erheblicher Teil des ostdeutschen Produktivvermögens im Besitz von Westdeutschen ist und daB bis heute und auch noch in absehbarer Zukunft weitaus mehr Waren und Dienstleistungen in das Gebiet der ehemaligen DDR ausgeführt als umgekehrt von dort bezogen werden; daher landet ein beträchtlicher Teil der für Ostdeutschland bestimmten Finanztransfers letztendlich wieder im Westen. 
Zugleich spricht aber sehr vieles dafür, da B gerade die ehemalige DDR doch rascher als die meisten anderen Länder des früheren RGW den AnschluB an die wirtschaftliche Leistungsfähigkeit der fortschrittlichsten Industrienationen finden wird, obwohl sie durch die schlagartige Währungsunion vom 1. Juli 1990 und die politische Vereinigung vom 3. Oktober desselben Jahres, ausgedrückt in den Kategorien der Wirtschaftswissenschaft, eine "Schocktherapie“ par excellence erlebt hat und obwohl sie trotz ihrer vergleichweise günstigen Ausgangsbedingungen besonders hart vom Tansformationsproze $B$ betroffen war. jedenfalls was den Umfang der industriellen Produktion und den Verlust profitabler Arbeitsplätze anging. Für diese letztendlich positive Einschätzung sprechen vor allem folgende Gesichtspunkte:

- Die massiven Finanztransfers von West nach Ost werden wenigstens teilweise auch in den Kauf heimischer Produkte in den neuen Bundesländern gehen, nachdem westliche und westdeutsche Waren ihren anfänglichen Exotenbonus verloren haben und von den Konsumenten nüchterner auf Preis und Qualität hin betrachtet werden. Auch die anfängliche "Auslistung" ostdeutscher Waren durch die neuen westdeutschen Eigentümer im Einzelhandel der ehemaligen DDR scheint inzwischen, schon aus nüchternem ökonomischen Kalkül, beendet worden zu sein.

- Die gewaltigen öffentlichen Investitionen, insbesondere im Infrastrukturbereich (Verkehrswege aller Art, Versorgungsleitungen, Kläreinrichtungen usw.), werden ebenfalls eine stabilisierende Wirkung entfalten, da sie wichtige Produktionsvoraussetzungen schaffen und überdies ja auch auf längere Zeit angelegt und beschäftigungswirksam sind.

- Der wichtigste Grund aber für die Erwartung einer besonders günstigen Entwicklung in der ehemaligen DDR ist die Tatsache, daß diese mit dem Einigungsvertrag im wesentlichen das gesamte westdeutsche Rechts- und Institutionensystem übernommen hat und damit auf eine - wenn auch sehr ngrobschlächtig" erscheinende - Weise einen institutionellen. rechtlichen und politischen Ordnungsrahmen für den ökonomischen Transformationsproze $B$ erhalten hat. Selbst die Tatsache, da $B$ angesichts der überstürzten Vereinigungsverhandlungen dieser "Ordnungsrahmen« mehr mübergestülpt» als wirklichd frei vereinbart und nicht in hinreichend differenzierter Weise transferiert worden ist, ändert wenig daran, da $B$ die Ubernahme dieser äußeren Bedingungen zusammen mit der Úbernahme der international als stark anerkannten D-Mark quasi auf einen Schlag ordnungspolitische Rahmenvoraussetzungen geschaffen hat, über die keines der anderen Reformländer Mittel- und Osteuropas auch nur annähernd verfügt. Diese müssen ihren Ordnungsrahmen erst selber schaffen und versuchen, ihre eigenen Währungen in einem mühseligen und vielfach blockierten Prozeß der Integration in die weltweite Arbeitsteilung auf den internationalen Finanz- und Devisenmārkten zur Geltung zu bringen.

Insbesondere im Hinblick auf die damals bevorstehende deutsch-deutsche Einigung wurde im Winter und Frühjahr 1990 häufig die westdeutsche Währungsreform 1948 zum Ausgangspunkt für vergleichende Überlegungen und Mutmaßungen über die Entwicklung in Ostdeutschland, aber auch in den anderen RGW-Ländern gemacht; wie wir oben gesehen haben, haben sich darauf gegründete optimistische Prognosen nicht erfüllt. Dies hängt zum einen vor allem damit zusammen, da $B$ trotz mancher Gemeinsamkeiten auch wesentliche Unterschiede zwischen 1990 und 1948 bestanden ${ }^{4}$. Sie sind vor allem in folgenden Punkten zu sehen:

- Mindestens innerhalb Deutschlands ist seit 1990 eine unbehinderte und mit geringen Transaktionskosten belegte Migration von Arbeitskräften (typischerweise vom Osten in den Westen) möglich; eine derartige Situation war nach dem Zweiten Weltkrieg noch nicht einmal zwischen den Ländern 
gegeben, die am Marshallplan beteiligt waren, geschweige denn, daß eine wesentliche Migration zwischen RGW- ur.d Nicht-RGW-Ländern möglich gewesen wäre, wenn man einmal von der Abwanderung aus . $3 Z$ und DDR in die Bundesrepublik bis zum Bau der Mauer im August 1961 absieht.

- Auch wenn nicht nur in Deutschland, sondern in nahezu allen europäischen Ländern w ährend des Zweiten Weltkrieges planwirtschaftliche Verhältnisse und Regelungen eingeführt worden waren, so waren doch Privateigentum, Vertragsfreiheit und unternehmerische Entscheidungsfreiheit wenigstens teilweise erhalten geblieben oder doch zumindest im Bewußtsein vieler Beteiligter noch existent.

- Im wesentlichen waren auch die privatwirtschaftlichen Akteure, jedenfalls in den westlichen Besatzungszonen Deutschlands, trotz der Ereignisse des Krieges und der unmittelbaren Nachkriegszeit mehr oder weniger unverändert geblieben; selbst die Neubildung der 1933 verbotenen Gewerkschaften ließ sich noch aus einer unmittelbaren Tradition und Erinnerung vieler Beteiligter speisen und bewerkstelligen. Demgegenüber fehlte eine derartige "Akteursidentität « in den meisten RGW-Ländern, die DDR hier eingeschlossen, weitgehend und im Falle der Sowjetunion praktisch völlig. Die mutigen ordnungspolitischen Entscheidungen nach dem Krieg. die mit den Namen Eucken, Müller-Armack und Erhard verbunden sind - insbesondere der systematische Abbau kriegswirtschaftlicher Reglementierungen -. stießen also auf Akteure, die in der Regel noch unmitttelbare marktwirtschaftliche Erfahrungen besaßen.

- Hinzu kam. daß die für die Gestaltung der ökonomischen Beziehungen besonders wesentlichen Rechtsgebiete, wie Bürgerliches Recht, Handelsrecht. Gesellschaftsrecht usw., vom Nazi-Regime kaum angetastet worden waren. Auch in dieser Beziehung war die Situation in den ehemaligen RGW-Ländern 1989/90 wesentlich verschieden: Zum Teil waren die entsprechenden Rechtsvorschriften nach kommandowirtschaftlichen Erfordernissen umgestaltet worden; aber auch da, wo sie formal in Kraft geblieben waren, hatten sie häufig durch seltene oder gänzlich fehlende Anwendung ihre normative Krait im Bewußtsein der Beteiligten verloren. Dies zeigte sich besonders deutlich in der Spätphase der DDR 1989/90, als man entdeckte, daß viele Vorkriegsregelungen des Wirtschaftsrechts zwar formal gültig geblieben, aber doch faktisch kaum mehr angewendet worden waren. Dies verschärfte noch die Probleme, die sich für die RGW-Länder aus der Tatsache ergaben. $\mathrm{da} B$ viele ökonomische Akteure gar keinen unmittelbaren Bezug mehr zu marktwirtschaftlichen Traditionen und Erfahrungen hatten.

\section{Probleme der Rechts- und Staatsordnung in den anderen RGW-Ländern}

Diese Überlegungen über Kontinuität und Bruch von Lebensordnungen und Bewußtseinsformen verweisen auf die Notwendigkeit, die soziokulturellen Bedingungen des Wirtschaftens, und damit auch ökonomischer Transformationsprozesse, näher in den Blick zu nehmen, als dies die traditionelle ökonomische Transformationsdebatte tut, die sich mehr um sinnerökonomische « Probleme - wie etwa die zum Glaubenskrieg hochstilisierte Frage nach der Überlegenheit einer "Schocktherapie" im Vergleich zu einem "graduellen Übergang" Gedanken macht als um die mindestens ebenso wichtige (und gerade auch für Reformstrategier relevante) Frage des Zusammenhangs verschiedener Lebensbereiche. Wie bereits 
erwähnt, lassen sich aus den ökonomischen Daten bisher keine zweifelsfreien empirischen Aussagen über die tatsächliche Überlegenheit einer der beiden Transformationsstrategien ableiten. Ich komme darauf nochmals zurück.

Unsere Überlegungen deuten ohnehin darauf hin, daß es nicht nur auf die Gestaltung der ökonomischen Prozesse. sondern auch und vor allem auf die Gestaltung der ökononischen Ordnung ankommt, und dies in Abstimmung mit anderen - staatlichen, rechtlichen, kulturellen usw. - Lebensbereichen. Bevor ich abschließend auf diese Frage eingehe. möchte ich noch kurz die Bereiche benennen und knapp kommentieren, in denen sich nach vorherrschender Überzeugung (Fischer, 1993, S. 390) Erfolg oder Mißerfolg des Transformationsprozesses entscheiden wird:

1. Makroökonomische Stabilisierung,

2. Preisfreigabe und Marktreform,

3. Unternehmensreform,

4. Handelsliberalisierung und Währungskonvertibilität,

5. Schaffung eines Netzes sozialer Sicherheit und

6. Schaffung eines rechtlichen und institutionellen Rahmens für eine Marktwirtschaft.

Bemerkenswerterweise wird bei dieser Aufstellung die Schaffung des rechtlichen und institutionellen Rahmens, die in der Tat Voraussetzung und Erfolgsbedingung für alle übrigen Maßnahmen ist, erst an letzter Stelle erwähnt, und die gesellschaftlichen und kulturellen Bedingungen für das erfolgreiche Funktionieren marktwirtschaftlicher Beziehungen werden erst gar nicht genannt. Wie wichtig aber gerade diese vier letztgenannten Bedingungen sind, das zeigt schon ein elementarer Vergleich zwischen dem Erfolg der Freigabe vieler Preise durch Ludwig Erhard im Zusammenhang mit der Währungsreform 1948 und dem relativen Mißerfolg derselben Maßnahme in den meisten Nachfolgestaaten der Sowjetunion. Während damals die von der ökonomischen Theorie prognostizierten Wirkungen in den meisten Bereichen rasch eintraten - die Preissteigerungstendenzen hieiten sich in Grenzen, und die Produktion weitete sich rasch aus, natürlich auch begünstigt durch eine bereits vorher betriebene Hortung in Erwartung der bevorstehenden Währungsumstellung -, ist eine entsprechende Angebotsausweitung auf den russischen Märkten kaum zu beobachten: Es gibt zwar jetzt fast alles zu kaufen, aber zu Preisen, die für die Masse der Bevölkerung unbezahlbar sind. In der Sprache der ökonomischen Theorie: Die Preisfreigabe hat zwar der Rationierungsfunktion der Preise zum Durchbruch verholfen, ihre Allokationsfunktion - die Tendenz, Faktoren in profitable Verwendungsrichtungen zu lenken und so zu einer Produktionsausweitung beizutragen - hat sie jedoch bisher kaum zur Geltung bringen können. Der Grund dafür ist denkbar einfach: Während in einer funktionierenden Rechtsordnung kurzfristige Preissteigerungen aufgrund von Güterknappheit zugleich Anreize für mittel- und längerfristige Produktionsausweitungen aufgrund gestiegener Profitchancen bieten, kann dieser Mechanismus unter dem gegenwärtigen russischen Bedingungen kaum wirken. Abgesehen von allen wichtigen Mentalitätsfragen (vgl. Uhlig/Büscher, 1993) verhindern ganz vordergründige Faktoren, wi: monopolistische Preisabsprachen und die Kontrolle des Marktzugangs durch mafiaähnliche Organisationen, daß es zu den gesellschaftlich erwünschten und 
von der Theorie prognostizierten Angehotsausweitungen und den damit verbundenen Preissenkungstendenzen kommt. Hier zeigt sich ganz unmittelbar die Bedeutung einer funktionierenden Rechts- und Staatsordnung für das von der Theorie erwartete Effizienzpotential der Marktwirtschaft.

Selbst wenn man den oben angedeuteten ökonomischen Engführungen folgt, zeigt sich bereits ein Problem darin, daß die sechs genannten Problemfelder sehr eng wechselseitig miteinander verbunden sind, so da $B$ häufig ausgeklügelte Transformationsstrategien nicht zu organischen Entwicklungen, sondern vielmehr zu "circuli vitiosi“ führens: Die Freigabe der Preise und die notwendigen Marktreformen stoßen notwendig auf Widerstand und behindern die erforderliche Unternehmensreform durch den Widerstand von Managern und Arbeitern, die allen Anlaß zu der Befürchtung haben, daß sie aufgrund der Preisliberalisierung ihre Arbeitsplätze verlieren werden, ohne da $B$ schon ein adäquates soziales Netz aufgebaut ist, das ihnen im Falle der Arbeitslosigkeit wenigstens die Deckung ihrer Grundbedürfnisse ermöglicht. Die auch aus diesen Gründen geringe Flexibilität in der Anpassung ihrer Produktion verschärft aber zusätzlich die noch jeder Freigabe inhärenten Preissteigerungstendenzen und damit über eine allgemeine Teuerung auch die bereits bestehenden sozialen Probleme. Die erforderliche makroökonomische Stabilisierung scheitert oft daran. daß nicht nur der erforderliche ökonomische Sachverstand, sondern auch die notwendigen staatlichen Finanzmittel fehlen, die in einer Zeit schrumpfender Produktion dramatisch zurückgehen. verschärft noch dadurch, da $B$ Versuche mancher Reformländer, wie Ungarns, durch Steuersenkungen und Zollabbau ausländisches Kapital zu attrahieren, weitere Löcher in die öffentlichen Haushalte reißen. Staatliches Handeln setzt überdies handlungsfähige staatliche Akteure voraus, eine Voraussetzung, die insbesondere in zahlreichen GUS-Staaten als äußerst zweifelhaft erscheinen muß.

Das marktmäßige "System der natürlichen Freiheit», wie Adam Smith (1776) es entworfen hat, setzt eben bereits entwickelte Institutionen, etablierte Handelsbeziehungen und nicht zuletzt die Entwicklung von Bewußtsein, Wertvorstellungen und Handlungsweisen voraus, welche die Institutionen. Normen und Regeln des Marktsystems im wesentlichen als gegeben betrachten, sei es. weil sich ein kurzfristiger Vertrauens- und Vertragsbruch in Erwartung langfristiger wechselseitig vorteilhafter Austauschbeziehungen überhaupt nicht lohnt oder sei es aus minnerer Überzeugung ". Häufig wird man in real existierenden Marktwirtschaften eine Mischung aller drei Motivationsgrundlagen feststellen können, die sich dann wechselseitig stabilisieren. Umgekehrt gilt natürlich, daß der Zusammenbruch der staatlichen Ordnung im Transformationsprozeß normenerodierend wirkt, weil die Akteure in einer Situation, in der nicht das Recht des ehrbaren Kaufmanns, sondern das Recht des Stärkeren gilt, die Erfahrung machen, daB Rechtstreue sich nicht lohnt, ja sogar, daß es sich lohnt, nicht rechtstreu zu sein. Hier können simultane Zusammenbrüche der wirtschaftlichen, politischen und geistigen Ordnungen eintreten. Inwieweit derartige Destabilisierungen durch "Markt- und Staatssurrogate«, wie Schattenwirtschaft, personale Beziehungen, spezialisierte Informationsmärkte, private Ordnungskräfte, aufgefangen werden können, bleibt fraglich: aber selbst wenn dies längerfristig gelingen sollte, binden derartige Surrogate erhebliche Ressourcen, die dann für den wirtschaftlichen Aufschwung fehlen. 


\section{Marktwirtschaft und die Interdependenz der Ordnungen}

Die Idee der Marktwirtschaft, so wie sie Adam Smith begründet und in unserem Jahrhundert vor allem Walter Eucken weiterentwickelt hat, besteht ja gerade darin, daB die wirtschaftlichen Akteure sich an die gegebenen Bedingungen des Konkurrenzmarktes anpassen und so gezwungen werden, ihren Eigennutz in gesellschaftlich nützliche Bahnen zu lenken. Was sich dagegen in vielen Reformländern des Ostens abspielt, ist der umgekehrte Versuch: Das Fehlen etablierter Werte, die Schwäche vieler staatlicher Ordnungen und auch oft das verständliche Streben, sich unmittelbarer Not zu entziehen, führen dazu, daß sich einzelne Akteure oder Gruppen mit dem Recht des Stärkeren die ökonomischen und politischen Bedingungen zu ihrem eigenen Vorteil zurechtzubiegen versuchen, sie sich anzupassen suchen. Wohin das auch in ökonomischer Hinsicht führen kann, habe ich oben am Beispiel mafiaähnlicher Marktbeherrschung dargestellt. Besonders problematisch sind dabei zwei Aspekte: Zum einen wird die Etablierung einer stabilen, verläßlichen Rechts- und Staatsordnung aufgrund ständiger gruppen- und personenspezifischer Interventionen zusätzlich erschwert, und zum anderen sind unter derart ungesicherten Ordnungsbedingungen vor allem die für wirtschaftliche Entwicklung zentralen langfristigen Transaktionen - besonders die Investitionen - weitgehend blockiert.

Die ökonomische Theorie und die ihr entsprechende wirtschaftspolitische Praxis ist - au. verständlichen Gründen, denn sie ist im wesentlichen als theoretische Reflexion und staatliche Absicherung bereits eingetretener ökonomischer Aktivitäten entstanden - auf das Problem der Transformation denkbar schlecht vorbereitet. Schon vor 150 Jahren hatte Karl Marx (1847, S. 126), dessen ökonomisches Denken im Zuge der jüngsten dramatischen Veränderungen in Verruf und Vergessenheit zu geraten scheint, doch immerhin die Nationalökonomen seiner Zeit zu Recht dafür kritisiert, daß sie zwar erklären, wwie man unter den ... gegebenen Verhältnissen produziert; was sie aber nicht erklären, ist, wie diese Verhältnisse selbst produziert werden, das heißt die historische Bewegung, die sie ins Leben ruft «. Selbst in enger ökonomischer Perspektive stellen sich heute Fragen, für die die ökonomische Theorie keine unmittelbaren Antworten hat. So erfordert ein einheitliches Wirtschafts- und Staatsgebiet tendenziell einheitliche Güter- und Faktorpreise, also auch Löhne und Gehälter; diese lassen sich unter marktwirtschaftlichen Bedingungen aber auf Dauer nur sichern, wenn eine entsprechende Poduktivitätsentwicklung stattfindet.

Dies betrifft vor allem das vereinte Deutschland: Während einerseits eine zu langsame Anpassung der Ost-Löhne an das West-Niveau die ohnehin schon bestehenden Migrationstendenzen verstärkt und gerade zum Verlust derjenigen qualifizierten und mobilen Arbeitskräfte führt, auf die der Aufschwung in den neuen Bundesländern so dringend angewiesen ist, kann andererseits eine zu rasche Angleichung. wie sie von vielen Tarifpartnern vereinbart wurde, ebenfalls den Aufschwung abdrosseln, Arbeitsplätze vernichten und so die Migration ihrerseits fördern, nämlich über den vermeidbaren Konkurs vieler Unternehmen und vor allem durch lohnkostenbedingte Barrieren gegen den Aufbau neuer produzierender Einheiten und damit neuer Arbeitsplätze. Hier kann nicht die Theorie, sondern nur der praktische Menschenverstand weiterhelfen. Konkret würde dies in diesem Falle bedeuten, daß man einerseits den Zeitraum für die Lohnangleichung streckt (auf 8-10 anstatt 5 Jahre), aber 
andererseits den Menschen in den neuen Bundesländern die Perspektive und Zusage gibt, da $B$ diese Lohnangleichung dann duch wirklich stattfindet. Hier ist dänn vor allem Vertrauen gefordert. eine Ressource, die der ökonomische Proze $B$ aus sich allein heraus nicht zu erzeugen vermag. ja. die er sogar immer wieder zu zerstören tendient ${ }^{6}$.

Ich möchte kurz anoeuten, wie die prinzipiellen Überlegungen der "Väter der Sozialen Marktwirtschaft « nach dem Zweiten Weltkrieg, von Walter Eucken (1940, 1951, 1952), Alfred Müller-Armack (1949a, 1949b, 1974) und Ludwig Erhard, trotz der erwähnten Unterschiedlichkeit in der Ausgangslage so eingesetzt werden können, da $B$ sie die erforderlichen soziokulturellen Bedingungen für einen gelingenden Transformationspropze $B$ schaffen helfen. Dazu möchte ich in ganz komprimierter Form die Grundgedanken von Euckens Ordnungspolitik wiedergeben?

Sein Ansatz ist als Versuch zu verstehen, die Schwächen des klassischen Liberalismus und die damit verbundenen wirtschaftspolitischen Erfahrungen bis zur Mitte des 20. Jahrhunderts (wie Konzentration und Monopolisierung, die deutsche Hyperinflation 1923, die Weltwirtschaftskrise 1929-1934) produktiv aufzunehmen und in die liberale ökonomische Theorie zu integrieren. Eucken (1952. S. 373) erkennt richtig, daß sich die auch von ihm gewünschte Wettbewerbsordnung nicht von selbst als Smith' "natürliches System der Freiheit « herausbildet. so da $B$ es eben nicht ausreicht, "gewisse Prinzipien des Rechts zu verwirklichen und im übrigen die Entwicklung der Wirtschaftsordnung sich selbst zu überlassen«. Es besteht also staatlicher Handlungs- und Gestaltungsbedarf - es braucht eine spezifische Ordnungspolitik -, damit ndie starken Tendenzen zur Wirkung [kommen], die auch in der industriellen Welt zur vollständigen Konkurrenz drängen«. Für Eucken st daher Wirtschaftsordnung nicht nur historisch vorfindbare Ordnung. sondern zugleich eine auf analytischer Grundlage beruhende und gestaltete "sinnvolle Zusammenfügung des Mannigfachen zu einem Ganzen«, denn: "Der Mensch will wissen, wie die konkreten Ordnungen sind, und er sucht eine bessere Ordnung" (1952, S. 372).

Die richtige Wirtschaftsordnung ist daher eine Gestaltungsaufgabe, bei der Interdependenz in einem doppelten Sinne zu beachten ist: Zum einen erscheint sie als wechselseitige Abhängigkeit waller wirtschaftlichen Erscheinungen, aller Bewertungen, aller Handlungen«, und zum anderen als wechselseitige Abhängigkeit der Wirtschaftsordnung insgesamt wie auch ihrer Teilordnungen mit allen üt rigen (staatlichen, gesellschaftlichen, kulturellen usw.) Ordnungen. Entscheidend ist dabei Verläßlichkeit der Rahmenbedingungen des Wirtschaftens durch deren langfristig konzipierte aktive staatliche Gestaltung und Gewährleistung bei weitgehendem Verzicht auf punktuelle. interventionistische Eingriffe in den WirtschaftsprozeB. Die Konstanz der Wirtschaftspolitik ist daher ebenso wichtig wie der Vorrang der konstituierenden Prinzipien vor den stärker situationsspezifisch bedingten regulierenden Prinzipien. Es ist ein bleibendes - und für die Transformationsprozesse bisher viel zu wenig genutztes - Verdienst Euckens, eine klare und systematische ordnungspolitische Konzeption entwickelt zu haben, die sich zugleich erforderlichem Handeln im Einzelfall nicht um der Systematik willen verschließt.

Alfred Müller-Armack und Ludwig Erhard haben. von dieser Grundlage ausgehend, mit der Sozialen Marktw'irtschaft eine realtypische Wirtschaftsordnung angestrebt, in der wirtschaftliche Leistungsfähigkeit und soziale Sicherheit in ein immer gefährdetes, aber in der Geschichte der Bundesrepublik auch immer wieder erreichtes Gleichgewicht gebracht werden. Auf der 
Grundlage dieses Ausgleichs wird auch prinzipiell die Abstimmung des wirtschaftlichen Systems mit den anderen Lebensbereichen und Ordnungen möglich und aussichtsreich ${ }^{8}$. Nicht ökonomischer Imperialismus, der durch die Universalisierung des Wirtschaftlichen die geistigen und kulturellen Grundlagen untergräbt, sondern die sinnvolle Einbettung des Ökonomischen in die Gesamtheit aller Lebenszusammenhänge ist das eigentliche ethische Ziel des ordoliberalen Ansatzes. Hier kann - bei aller oben betonten Differenz der Jahre 1948 und 1990 - viel Grundsätzliches, aber auch manches Praktische für den Transformationsprozeß gelernt werden. vor allem im Hinblick auf ein problembezogenes Vorgehen. So wurde die Frage der Preisfreigabe nach dem Zweiten Weltkrieg zwar auf der grundsätzlichen Ebene eindeutig zugunsten eines Vorrangs dieser Maßnahme. also im Sinne einer generellen liberalen Richtungsbestimmung, entschieden; gleichzeitig wurden aber Übergangsfristen vor allem in den Bereichen. wie etwa dem Wohnungsbau, geschaffen, in denen aufgrund der Produktionsvoraussetzungen marktwirtschaftliche Anpassungsprozesse längere Zeit benötigten. Damals wurde die heutige Engführung von "Schocktherapie versus Gradualismus" vermieden zugunsten einer entschlossenen, aber auch problembezogenen und längerfristig konzipierten marktwirtschaftlichen Übergangsstrategie. Eine derartige Politik des langen Atems ist auch heute im vereinten Deutschland und noch mehr in den Reformländern Mittel- und Osteuropas wichtiger denn je. Ihre entscheidende Erfolgsvoraussetzung ist dabei Vertrauen der Menschen in die prinzipielle Unumkehrbarkeit des Reformweges und die konkrete Situationsbezogenheit der Reformschritte - also eben eine weitgehend außerökonomische, soziokulturelle Voraussetzung und Grundlage wirtschaftlichen Handelns ${ }^{9}$.

\section{Kulturelle, religiöse und ethische Grundlagen}

Die kulturellen und ethischen Grundlagen des Wirtschaftens wurden und werden, wie wir gesehen haben, von der Mehrzahl der Wirtschaftswissenschaftler, vor allem in den angelsächsischen Ländern, häufig deutlich unterschätzt, und diese ökonomische Engführung hat mit zum bisher höchst unbefriedigenden Verlauf des Transformationsprozesses in den Ländern Mittel- und Osteuropas beigetragen. Man darf allerdings nicht in den umgekehrten Fehler verfallen und die kulturell-ethischen Grundlagen des Wirtschaftens instrumentell als womöglich noch kurzfristigen - "Erfolgsfaktor " begreifen, den es einfach zu aktivieren gelte. Gleichwohl: Im Guten wie im Bösen wirken die soziokulturellen und geistigen Traditionen aller Länder und Völker auf ihre geistige, kulturelle und damit auch wirtschaftliche Lebenspraxis zurück. Von daher mag man im Hinblick auf Länder wie die Tschechische Republik oder Ungarn optimistischer urteilen, da diese nicht nur bis zur kommunistischen Machtübernahme weitgehend in die damalige weltwirtschaftliche Arbeitsteilung eingebunden waren, sondern darüber hinaus auch in engem geistigen und kulturellen Kontakt zu den fortgeschrittenen Industrieländern standen. Schwieriger, aber keineswegs unlösbar erscheinen in dieser Hinsicht Probleme in den südosteuropäischen Ländern, deren ökonomischer, aber auch kultureller Kontakt zu West- und Mitteleuropa schun vor 1945 weniger eng ausgeprägt war. Eine Sonderstellung dürfte hierbei ?olen zukommen, dessen traditionell enge kulturelle 
und intellektuelle Beziehungen zu Westeur spa nicht von einer entsprechend fortgeschritteren wirtschaftlichen Entwicklung und Koope:ation begleitet waren.

Die gegenu urtige politische und wirtschaftliche Entwicklung in zahlreichen Nachfolgestaaten der ehemaligen Sowjetunion deutet darauf hin, daß dort die kulturellen und institutionellen Grundlagen einer marktwirtschaftlichen Entwicklung besonders defizient sind (vgl. Uhlig Büscher 1993); hier paaren sich kulturelle Sonderentwicklungen, wie etwa die Tradition des russischen Idealismus, mit wirtschaftlicher Rückständigkeit in vielen Bereichen und dem Fehlen demokratischer und rechtsstaatlicher Traditionen. Derartige Grundlagendefekte können nicht kurzfristig instrumentell ausgeglichen werden, aber sie stellen auch keine unüberwindliche Entwicklungsschranke dar. Zum einen gibt es auch in Rußland und anderen GUS-Staaten durchaus Anknüpfungspunkte an eine gemeinsame europäische Kulturtradition, etwa in der Malerei und der Literatur, und in vielen Bereichen der Wissenschaft hat Rußland und späte: die Sowjetunion immer AnschluB an die internationale Entwicklung gehalten. Diese progressiven Elemente gilt es im Bewußtsein nicht nur der Wissenschaft und der intellektuellen, sondern auch der Bevölkerung generell zu stärken. Wichtiger aber noch ist die Vermittlung der Einsicht, daß Rechtsstaatlichkeit und damit der Verzicht auf kurzfristige opportunistische Sondervorteile einzelner Gruppen und Akteure zentrale Voraussetzungen zur Aufhebung jenes "Gefangenendilemmas" sind, in dem sich derzeit vor allem die Gesellschaft und Wirtschaft Rußlands zu befinden scheinen.

Es mag sein, daß die "Erfahrung der Leiden«, die sich als zentrales Thema durch die russische und sowjetische Kulturgeschichte zieht, hier ein hemmendes Element darstellt; die gegenwärtigen Fehlsteuerungen des Transformationsprozesses in manchen Bereichen - wie Zerfall der alten staatlichen und politischen Ordnung ohne gleichzeitigen Aufbau neuer Ordnungsstrukturen, begleitet von Teuerung, Rechtsunsicherheit, Korruption und monopolitischer. Praktiken aller Art - lassen sich durchaus in diesem Kontext sehen. Aber auch diese soziokulturellen Faktoren - Stichwort: "Erfahrung der Leiden « - sind ihrerseits ebenfalls Ausdruck einer gesellschaftlichen und politischen Entwicklung in den letzten Jahrhunderten, die schon zuvor dem Aufbau einer modernen Wirtschaft nicht besonders zuträglich war. Die gegenwärtige Praxis des urent-seeking", also des Strebens nach Sondervorteilen zu Lasten der Allgemeinheit, ist Bestandteil eines Lebens- und Überlebenskampfes, der selbst durch diese Praxis verschärft wird. Die Schwierigkeit, alltägliches Leben unter diesen Bedingungen zu organisieren, wird aber andererseits der Erkenntnis förderlich sein, da $B$ es aussichtsreicher ist, sich selber an sinnvolle wirtschaftliche, politische und geistig-kulturelle Ordnungen anzupassen, als individuelle und gruppenspezifische Bemühungen auf das gesellschaftlich fragwürdige Ziel auszurichten, sich diese Ordnungen selber zum eigenen kurzfristigen Sondervorteil anzupassen, sie quasi auszunutzen - Versuche, die nur entsprechende konterkarierende Bemühungen anderer Akteure provozieren. Gerade diese Alltagserfahrung vieler Menschen in Rußland und anderen Staaten Osteuropas könnte die Einsicht fördern, um wieviel sinnvoller es ist. sich zunächst einmal der Schaffung gesellschaftlichen Reichtums unter geordneten und sozial erträglichen Bedingungen zuzuwenden, als alle Kräfte auf einen regellosen. vom Recht des Stärkeren bestimmten Verteilungskampf um einen schrumpfenden Wohlstand zu bündeln. Diese Grundeinsicht findet sich auch in den religiösen Traditionen der 
betroffenen Völker - nämlich dem Chrsitentum und dem Islam -, und auch von daher könnte der Übergang in einen sinnvollen Transformationsprozeß kulturell gestützt werden.

Eine wichtige soziokulturelle Frage muß ich - die Grenzen meiner wirtschaftswissenschaftlichen Disziplin respektierend - offen lassen, die Frage nämlich, inwieweit eine marktwirtschaftliche Entwicklung an spezifische religiöse Voraussetzungen - Stichwort ist hier Max Webers "protestantische Ethik" - gebunden ist. Obgleich ich die christlich-westlichen Ursprünge der Marktwirtschaft weder leugnen will noch kann, bin ich doch als Ökonom optimistisch, daß langfristig auch andere religiöse Traditionen sich marktwirtschaftlichen Gedanken öffnen werden; zum Teil haben sie das auch schon getan, besonders in Ostasien, aber auch im säkularisierten Islam der Türkei. Einige der ostasiatischen Konkurrenten. gerade unter den vielzitierten "kleinen Tigern«, wären noch vor wenigen Jahrzehnten als ernsthafte Aspiranten auf eine erfolgreiche Weltmarktintegration belächelt worden. $\mathrm{Zu}$ bedenken ist aber vor allem eines: Es geht ja gar nicht um die schlichte Übernahme eines westlichen Ordnungsmodells, sei es angelsächsischer, sei es mitteleuropäischer Prägung; diese wäre in der Tat kaum zu leisten. Sie ist aber auch nicht notwendig, denn die Marktwirtschaft erfordert zwar einige Grundprinzipien wie Vertragsfreiheit und Eigentumsschutz, ist aber im übrigen ein durchaus flexibles Konzept, das sich in unterschiedlicher Weise in jeweils sinnvolle Abstimmung mit spezifischen kulturellen und historischen Traditionen bringen läßt; Japan hat tafür das erste, aber keineswegs das letzte Beispiel gegeben. Allerdings zeigen die Erfainrungen real existierender Marktwirtschaften in verschiedenen Teilen der Welt, daß sie ähnlich wie die jetzt zugrundegegangenen Kommandowirtschaften - immer dazu tendieren. durch ihre Dynamik die Grundlagen des Wirtschaftens zu untergraben. Hier besteht für Industrie-, »Reform«- und Entwicklungsländer gleichermaßen eine zentrale Gestaltungsaufgabe für die nächste Zukunft.10

Worauf es vor allem ankommt, das ist die Möglichkeit für die Reformländer Mittel- und Osteuropas, konkrete positive Erfahrungen in und mit der Marktwirtschaft zu machen. Diese sind wichtiger als alles andere dafür, da $B$ sich die erforderlichen Normen und Werthaltungen heranbilden und dann an die kommenden Generationen weitergegeben werden können. Vertrauen und Verläßlichkeił als unabdingbare Voraussetzungen einer ökonomisch erfolgreichen und sozial verantwortlichen Marktwirtschaft können nur so heranwachsen und sich festigen. Der Westen könnte viel zu solch entscheidenden positiven Ökonomie-Erfahrungen beitragen, wenn er durch gezielte Hilfe (Transfer von Wissen, Qualifikationen. Technologie und Geld) und einen jeweils an der Leistungsfähigkeit orientierten partiellen Schuldnererlaß an dem Abbau unmittelbarer Not mitwirkt, die wenig geeignet ist, den Menschen in den ehemals kommunistisch-planwirtschaftlichen Staaten die Botschaft vom Reichtum der Nationen glaubhaft darzustellen.

Prof. Dr. Hans G. Nutzinger

Universität GH-Kassel

Fachbereich Wirtschaftswissenschaften - 34109 Kassel 


\section{Abstract}

Hans Nutzinger describes the current complex of problems of the eastern european countries on their transition to market conomy and points out. how far socio-cultural problems determine the conditions of transformation. The appropriate economic system seems to appear as a responsibility of organization, where as interdependence has to be seen in a double sense: as an interdependence of all economic decisions, valuations and actions and, on the other hand, as an interdependence of economic systems as a whole. He deals with the topic how far a free-enterprise development depends on specific religious preconditions, but leaves the question - respecting the limits of the economic discipline - undecided.

\section{Anmerkungen}

1. Universität-GH Kassel, Fachbereich Wirtschaftswissenschaften. und Wissenschaftskolieg zu Berlin (Fellow des Akademischen Jahres 1992/93). - Für die hilfsreiche Vortrags- und Diskussionsmitschrift danke ich Herrn Mark Kuhrt (Berlin); für kritische Anmerkungen zu früheren Versionen bin ich Frau Dr. Christine Kunert (Berlin) und Dr. Hans Diefenbacher (Heidelberg) dankbar. Der vorliegende Beitrag ist die überarbeitete Fassung eines Vortrags, den der Autor am 15. Juli 1993 bei einer gemeinsamen Veranstaltung der Kulturstiftung Haus Europa und des Deutschen Komitees für kulturelle Zusammenarbeit in Europa e. V. in Berlin gehalten hat.

2. So droht insbesondere die in mehreren Reformländern immer noch steigende Auslandsverschuldung zu einem ernsthaften längerfristigen Hindernis für den Aufschwung zu werden. Beispietsweise stieg in den ersten vier Monaten des Jahres 1993 die ungarische Auslandsverdschuldung um 2.2 Mrd. S auf 23,6 Mrd. S. Der Schuldendienst Polens an inländische und ausländische Gläubiger erreichte 1993 rund 5,8 Mrd. S; die Staatsverschuldung Polens droht zu explodieren. wenn nicht ein erheblicher Teil (im Gespräch sind 30-50\%) seiner gegenwärtig $12 \mathrm{Mrd}$. $S$ betragenden Schulden gegenüber ausländischen Geschäftsbanken abgeschrieben wird (vgl. Transition, Zeitschrift der Weltbank. Bd. 4. Nr. 7, September 1993, S. 14).

3. Einer Meldung der Weltbank zufolge hat die industrielle Produktion Ungarns in den ersten sieben Monaten des Jahres 1993 im Vergleich zur selben Periode des Vorjahres um 2.5\% zugenommen; gleichwohl wird zum Ende des Jahres eine Schrumpfung jes realen Bruttosoziatproduktes um 2 bis $3 \%$ erwartet, wobei die Lebenshaltungskosten um 22 bis $23 \%$ gegenüber Jem Vorjahr steigen werden und das Zahlungsbilanzdefizit mehr als $2 \mathrm{Mrd}$. S betragen wird (nach Transition. Bd. 4 Nr. 7, September 1993. S. 17).

4. Vgl. dazu die Beiträge von Möller und Willgeroth in Wagener (1991).

5. Vgl. dazu auch den Beitrag von Barbara Krug in Wagener (1991).

6. Vgl. dazu Siegenthaler (1993), vor allem Kap. VIII "Verlust an Regelvertrauen als Konsequenz wirtschaftlicher Entwicklung".

7. Vgl. dazu ausführlicher und mit weiteren Literaturhinweisen Nutzinger (1993a).

8. Genau aus diesem Grunde halte ich auch die gegenwärtigen Versuche mancher liberaler Protagonisten in Deutschland, dieses Konzept wieder in Richtung auf eine paläoliberale "Ellenbogengesellschaft« zu verkürzen. auch im Interesse der marktwirtschaftichen Ordnung für kontraproduktiv.

9. Dabei muß man natürlich zugeben, daß Euckens Überlegungen zur Interdependenz der Ordnungen, vor allem auch im Hinblick auf Zentralverwaltungswirtschaften und deren Transformation in Verkehrswirtschaften, sehr allgemein geblieben sind. Vgl. dazu auch Klinger (1993), bes. den letzten Abschnitt.

10. Vgl. dazu Nutzinger (1993b).

\section{Literatur}

Eucken. Walter: Die Grundlagen der Nationalökonomie. Jena: G. Fischer 1940. 8. Aufl. Berlin/Heidelberg: Springer, 1965.

Eucken. Walter: Unser Zeitalter der Mißerfolge. Fünf Vorträge zur Wirtschaftspolitik. Tübingen: J. C. B. Mohr, 1951 .

Eucken, Walier: Grundsätze der Wirtschaftspolitik. Tübingen: J. C. B. Mobr, 1952. 5. Aufl. 1975.

Fischer. Stanley: "Socialist Economic Reform: Lesson of the First Three Yearsu, in: American Economic Review 83. No. 2 (Mai 1993), S. 390-395.

Klinger, Fred: "Soziale Konflikte und offene Gewaltu, in: Deutschland Archiv 26. Jg., H. 2. 1993, S. 147-161. 
Marx. Karl: Das Elend der Philosopie (1847). In: Marx-Engels-Werke (MEW). Band 4. Berlin: Dietz, 1977. S. $63-182$.

Müller-Armack. Alfred: Soziale Irenik. Manuskript. Münster, Dezember 1949(a).

Müller-Armack. Alfred: Diangnose unserer Gegenwart. Zur Bestimmung unseres geistesgeschichtlichen Standorts. Gütersloh: C. Bertelsmann Verlag. 1949(b).

Müller-Armack, Alfred: "Der Theorie-Gehalt der Sozialen Marktwirtschaftu. In: 25 Jahre voraus (Vorträge und Disk ussionen der 37. Tagung der Aktionsgemeinschaft Saziale Marktwirtschaft am 25. und 26. Juni 1973 in Bonn-Bad Godesberg) Ludwigsburg: Martin Hoch. 1974. S. 36-56.

Nutzinger. Hans $G$..: "Der Begriff Verantwortung aus ökonomischer und sozialethischer Sichtu. In: Karl Homann (Hg.): Aktuelle Probleme der Wirtschaftsethik (Schrifteh des Vereins für Socialpolitik. N. F. 211). Berlin: Duncker \& Humblot. 1992, S. 43-67.

Nutzinger. Hans G.: "Ordnungspolitik «. In: G. Enderle u. a. (Hg.): Lexikon der Wirtschaftsethik. Freiburg Basel Wien: Herder. 1993a. S. 784-794

Nutzinger. Hans $G$.: "Langzeitverantwortung im Umweltstaat aus ökonomischer Sicht: Zur Konzeption des nachhaltigen Wirtschaftensu. In: Gethmann. Carl-Friedrich: Kloepfer. Michael und Nutzinger, Hans G.: Langzeitverantwortung im Umweltstaat. Bonn: Economica. 1993b. S. 22-58.

Siegenthaler, Hansjörg: Regelvertrauen. Prosperität·und Krisen. Die UngleichmäBigkeit wirtschaftlicher und sozialer Entwicklung als Ergebnis individuellen Handelns und sozialen Lernens. Tübingen: J. C. B. Mohr, 1993.

Smith, Adam: Der Wohlstand der Nationen. Eine Untersuchung seiner Natur und seiner Ursachen (engl. Original 1776). München: Deutscher Taschenbuch Verlag, 1978.

Transition. The Newsletter about Reforming Economies (Zeitschrift der Weltbank). Band 4. Nr. 7, 1993.

Uhlig, Christine und Büscher, Martin: Systemtransformation und Homo oeconomicus. Institutionelle Voraussetzungen der Marktwirtschaft am Beispiel der Wirtschaftsgesinnung im russischen Kulturraum. Manuskript, Hochschule St. Gallen. Februar 1993.

Wagener. Hans-Jürgen (Hg.): Anpassung durch Wandel. Evolution und Transformation von Wirtschaftssystemen (Schriften des Vereins für Socialpolitik. Bd. 206). Berlin: Duncker \& Humblot, 1991. 